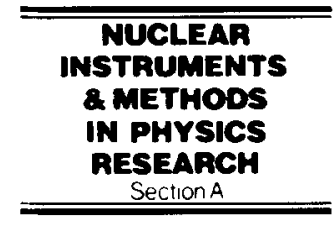

\title{
First lasing of TEUFEL ${ }^{\text {is }}$
}

\author{
G.J. Ernst ${ }^{a}$, J.W.J. Verschuur ${ }^{a}{ }^{*}$, B.M. Van Oerle ${ }^{b}$, A.F.M. Bouman ${ }^{b}$, J.I.M. Botman ${ }^{c}$, \\ H.L. Ilagedoorn ${ }^{\mathrm{c}}$, W.J. Witteman ${ }^{\mathrm{a}}$ \\ 'University of Twente, Dept. of Appl. Physics, P.O. Box 217, 7500 AE Enschede, The Netherlands \\ "Nederlands Centrum voor Laser Reseurch B.V., P.O. Box 2662, 7500 CR Enschede, The Netherlands \\ "Eindhoven University of Technology. Cycl. Lab., P.O. Box 513, 5600 MB Eindhoven. The Netherlands
}

\section{Introduction}

The TEUFEL experiment, a collaboration between the University of Twente, the Nederlands Centrum voor Laser Research (NCLR) B.V. and the Eindhoven University of Technology, lased for the first time on Wednesday. August 23, 1995. The electron beam is generated in a $1.3 \mathrm{GHz}$ photocathode RF linac operating at a nominal energy of $6 \mathrm{MeV}$ [1]. The distance between the micropulses is determined by the repetition frequency of $81.25 \mathrm{MHz}$ of the mode-locked $\mathrm{Nd}$ :YLF drive laser [2]. First lasing was obtained with the drive laser operating at an energy of $1.5 \mu \mathrm{J} /$ micropulse (corresponding to $2 \mathrm{nC} /$ bunch) with a FWHM of $20 \mathrm{ps}$ in the green. The hybrid undulator has a period of $25 \mathrm{~mm}$ and a peak field of $0.7 \mathrm{~T}$ [3]. The total number of periods $N_{\mathrm{u}}$ is 50 . An oscillator configuration was used when TEUFEL showed its first output.

\section{Configuration}

Because the electron beam is kept straight, the oscillator is quite unique as both mirrors of the optical cavity contain holes to transport the electron beam through. The vacuum tube inside the undulator has an inner diameter of $6 \mathrm{~mm}$. Since the wavelength of the generated light is about $200 \mu \mathrm{m}$, the tube acts as an overmoded waveguide. 'I he undulator is positioned asymmetrically in the optical cavity. In order to match the electron beam to the undulator strong focussing was required. Therefore a flat copper mirror with a $2 \mathrm{~mm}$ diameter hole was placed in the $6 \mathrm{~mm}$ diameter vacuum pipe as close as possible to the entrance of the undulator. The advantage of this is that the

This joint project has been supported (in part) by the Netherlands Technology Foundation (STW) and the Foundation for Fundamental Research on Matter (FOM).

*Corresponding author. E-mail: j.w.j.verschuur@tn.utwente.nl. holc could be made as small as possible. i.e. slightly bigger than the matched electron beam size at the entrance of the undulator. The total length of the cavity is $1.842 \mathrm{~m}$ and extends significantly beyond the undulator. Downstream. Downstream the undulator, the vacuum pipe is tapered over a length of $400 \mathrm{~mm}$ from a diameter of $6 \mathrm{~mm}$ to a diameter of $25 \mathrm{~mm}$ to allow for free transport of the electron beam through the optical cavity. The tapered section also avoids spurious reflection and allows the radiation to fill the waveguide by diffraction. A movable flat mirror with an $8 \mathrm{~mm}$ diameter hole is positioned at the down stream side of the tapered section. The hole diameter is chosen to have the same ratio to the waveguide diameter on both sides. The tuning range of the cavity is $8 \mathrm{~mm}$. After the cavity the electron beam and radiation field copropagate until they are separated by a $90^{\circ}$ spectrometer used to measure the electron beam energy spectrum. After the spectrometer the radiation is coupled out of the vacuum tube using a metal foil mirror and a poly-ethylene window. This setup allows simultaneous measurement of optical output and the kinetic energy spectrum of the beam.

\section{Demonstration of first lasing}

To avoid possible attenuation in air, the radiation is guided by an overmoded light pipe flowed with helium to the place where a pyroelectric detector is situated. For increased sensitivity, the pyroelectric detector had a slow time response and was not able to resolve the temporal behaviour of the macropulse. Lasing of TEUFEL was clearly demonstrated by the energy spectrum of the electron beam which showed a significant downward shift in mean energy accompanied by a broadening. Simultaneously, optical energy was registered by the pyroelectric detector. Fig. 1 shows two typical spectra of the complete macropulse for maximum radiation energy (a) and for the cavity detuned to have no optical output (b). These 


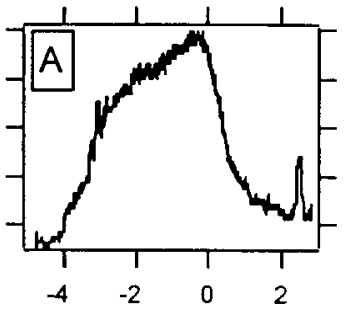

$\Delta \mathrm{E} / \mathrm{E}[\%]$

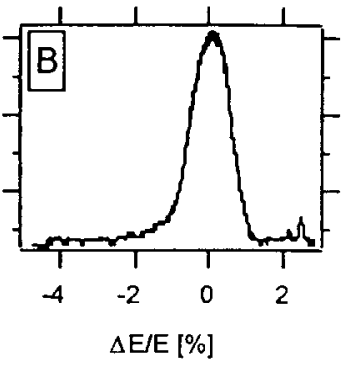

Fig. 1. Typical energy spectra of the macropulse for maximum (a) and no output (b)

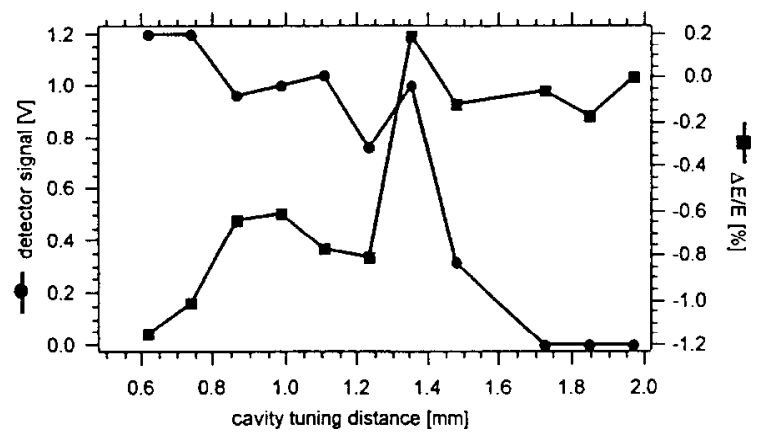

Fig. 2. Measured optical energy and a shift in mean kinetic energy as a function of change in cavity length. correspond to the cavity tuning values of 0.6 and $2.0 \mathrm{~mm}$ in Fig. 2 respectively. From these measurements the downward shift in mean energy is determined to be about $1.2 \%$ which indicates saturation. The saturation level is approximately $1 /\left(2 N_{\mathrm{w}}\right)$ (i.e. $1 \%$ ). The measured optical energy was less than expected from the above value, however the losses in the cavity (which include outcoupling at the upstream mirror) and transport system are unknown at this moment. Fig. 2 shows the optical energy and downward shift in mean kinetic energy of the electrons as a function of change in cavity length. The $8 \mathrm{~mm}$ tuning range did not allow for a full scan. For a detuning of around $1.4 \mathrm{~mm}$ the onset of lasing was observed, however, the output was rather unstable. Furthermore it was observed that apart from the first microsecond in the macropulse, the total optical energy was linear with the duration of the macropulse indicating a rather constant output power.

\section{References}

[1] G.J. Ernst et al., Infrared Phys. Technol. 36 (1995) 81.

[2] R.F.X.A.M. Mols and G.J. Ernst, Nucl. Instr. and Meth. A 341 (1994) 481.

[3] J.W.J. Verschuur, G.J. Ernst and W.J. Witteman, Nucl. Instr. and Meth. A 318 (1992) 847. 